\title{
Survival Trends After Surgery for Spinal Metastatic Tumors: 20-Year Cancer Center Experience
}

\section{Robert J. Rothrock, MD Ori Barzilai, MD $\mathbb{1 D}^{* \neq}$ Anne S. Reiner, MPH ${ }^{\S}$ Eric Lis, MD (1) \\ Adam M. Schmitt, MD" \\ Daniel S. Higginson, MD 에 Yoshiya Yamada, MD 에 \\ Mark H. Bilsky, MD ㅁ* \\ Ilya Laufer, MD $\mathbb{D}^{*}$}

*Department of Neurosurgery, Memorial Sloan Kettering Cancer Center, New York, New York; ${ }^{\ddagger}$ Department of Neurosurgery, Weil Cornell Medical College, New York, New York; ${ }^{\S}$ Department of Epidemiology and Biostatistics, Memorial Sloan Kettering Cancer Center, New York, New York; "Department of Neuroradiology, Memorial Sloan Kettering Cancer Center, New York, New York; "Department of Radiation Oncology, Memorial Sloan Kettering Cancer Center, New York, New York

\section{Correspondence:}

Ilya Laufer, MD,

Department of Neurosurgery,

Memorial Sloan Kettering Cancer Center, 1275 York Ave,

New York, NY 10065, USA.

Email: lauferi@mskcc.org

Received, November 1, 2019.

Accepted, June 27, 2020

Published Online, September 24, 2020.

Copyright (C) 2020 by the

Congress of Neurological Surgeons

BACKGROUND: Over the last 2 decades, advances in systemic therapy have increased the expected overall survival for patients with cancer. It is unclear whether the same survival benefit has been conferred to patients requiring surgery for metastatic spinal disease. OBJECTIVE: To examine trends in postoperative survival over a 20 -yr period for patients surgically treated for spinal metastatic disease.

METHODS: Data were obtained for 1515 patients who underwent surgery for metastatic epidural spinal cord compression or tumor-related mechanical instability. Postoperative overall survival was calculated for all included patients using Kaplan-Meier methodology from date of surgery until death or last follow-up for those who were censored. Trends were analyzed using Cox proportional hazards modeling.

RESULTS: Patients with renal, breast, lung, and colon cancers experienced a statistically significant improvement in survival over time based on the year of surgery $(40 \%-100 \%$ improvement over the study period), whereas the overall survival trend for the entire cohort did not reach statistical significance $(P=.12$, median survival $0.71 \mathrm{yr}, 95 \% \mathrm{Cl}$ 0.63-0.78). Patients presenting with synchronous metastatic disease had better survival compared to those presenting with metachronous disease (median overall survival: 0.94 vs $0.63 \mathrm{yr}$, respectively; log-rank $P$-value $=.00001$ ).

CONCLUSION: The postoperative survival among patients with spinal metastases has improved over the past $20 \mathrm{yr}$, particularly in patients with kidney, breast, lung, and colon tumors metastatic to the spine. The observed survival improvement emphasizes the need for long-term outcome consideration in treatment decisions for patients undergoing surgery for spinal metastatic tumors.

KEY WORDS: Survival for spinal metastases, Trends in survival, Separation surgery, Hybrid therapy

Neurosurgery 88:402-412, 2021 DOI:10.1093/neuros/nyaa380 www.neurosurgery-online.com

$\mathbf{0}$ ver the last 2 decades, advances in treatment and early diagnosis have increased the expected overall survival for patients with cancer. ${ }^{1}$ The 5 -yr relative survival among patients diagnosed with cancer improved from $35 \%$ in 1950 to 1954 to $70 \%$ for the 2009 to 2015 time interval. ${ }^{2}$ However, these composite survival statistics include patients with local, regional, and metastatic stages of cancer, with less information available for the survival trends specific to patients with metastatic cancer.

ABBREVIATIONS: CI, confidence interval; OS, overall survival; RPA, recursive partitioning analysis

Supplemental digital content is available for this article at www.neurosurgery-online.com.
The spine represents one of the most common sites of metastatic disease. Decisions regarding treatment strategy selection require consideration of the expected survival. Although the general cancer statistics indicate that patients diagnosed with cancer live longer, it is unclear whether the same survival benefit has been conferred to patients requiring surgery for metastatic spinal disease because these patients present at various stages of treatment. ${ }^{3}$ As more therapeutic agents and potential salvage therapies become available for patients with metastatic cancer, it has been assumed that longer overall survival for cancer patients has translated into longer postoperative survival, leading to greater emphasis on the maintenance of quality of life (QOL) and durable local tumor control in the treatment of spinal metastatic disease. ${ }^{4-8}$ 
The current study examined trends in survival over $20 \mathrm{yr}$ for patients undergoing surgery for the treatment of spinal metastatic tumors at a single institution. We hypothesized that with advances in systemic cancer therapy and surgical care, there would be a temporal trend toward improved survival based on the year of treatment.

\section{METHODS}

\section{Patient Selection}

All patients undergoing surgical intervention for metastatic spinal disease at Memorial Sloan Kettering Cancer Center from January 1998 to December 2017 were queried. The analysis was conducted in March of 2019. The gap between December 2017 and March 2019 provided at least 1 -yr follow-up interval for the most recently treated patient. The study was conducted under the supervision of the Institutional Review Board (MSKCC), and a patient consent waiver was granted given the retrospective nature of the study. The NOMS decision framework was utilized for the entire cohort. The NOMS framework consists of neurologic, oncologic, mechanical, and systemic considerations and is used to select the optimal combination of surgical, radiation, and systemic therapy for patients with spinal metastases. ${ }^{9}$ The primary surgical indications included high-grade metastatic epidural spinal cord compression and/or mechanical instability. Hybrid therapy consisting of separation surgery and radiotherapy was utilized for all patents requiring decompression of the spinal cord. Among patients requiring spinal stabilization, instrumentation placement was carried out using posterior approach for open or minimally invasive surgical stabilization. All patients had a clear surgical indication directly related to a metastatic cancer diagnosis and all had spinal instrumentation. All patients underwent radiation therapy using conventional external beam or stereotactic body radiation therapy. Patients were included only if the pathology examination of the surgical sample demonstrated viable metastatic disease. For patients who had multiple surgical interventions, the first surgery was used as the index surgery. The study did not exclude patients based on the duration of follow-up. Patients less than $18 \mathrm{yr}$ of age at the index surgery were excluded. Patients were excluded if they underwent only kyphoplasty or percutaneous needle biopsy without separation surgery or spinal instrumentation. Patient selection is presented in Figure, Supplemental Digital Content.

For patients who met the inclusion criteria, multiple variables were collected including age at index surgery, sex, primary cancer histology, pathology of metastatic lesion, synchronous or metachronous disease at presentation, level of spinal lesion, surgery performed, date of last followup, and patient vital status. Synchronous disease was defined as surgery on spinal metastasis within 3 mo of primary cancer diagnosis. Further analysis was performed for primary cancer sites representing greater than $4 \%$ of the overall cohort.

\section{Statistical Analysis}

Descriptive statistics such as frequencies, means, and ranges were used to characterize the cohort under study. Overall survival was defined as the time from index surgery for spine metastases until date of death or date of last known follow-up for those who were censored. KaplanMeier methodology was used to graphically display overall survival and for calculation of median overall survival. The log-rank test was used to compare overall survival experiences by categorical variables of interest.
To examine the temporal trends of survival, Cox modeling was used to associate calendar year of surgery with survival for the overall cohort and by primary cancer sites of interest. To identify possible splits of calendar time and their association with survival, recursive partitioning analysis (RPA) was performed for the overall cohort and by primary cancer sites of interest. ${ }^{10}$ To examine changes in 30- and $90-\mathrm{d}$ mortality over time, logistic modeling was used to associate calendar year of surgery with 30 - or $90-\mathrm{d}$ mortality for the overall cohort and by primary cancer sites of interest. Statistical tests were 2-sided with an alpha level of statistical significance set at 0.05 . All analyses were performed using SAS version 9.4 (SAS, Cary, North Carolina) and R software version 3.5.2 (in particular, the RPART package; RStudio).

\section{RESULTS}

The analysis includes 1515 patients who met the inclusion criteria (Figure, Supplemental Digital Content). The mean age at index surgery was 60 (range 18 to $92 \mathrm{yr}$ ), with males representing $60 \%$ of the study population (Table 1). Overall median follow-up for survivors was 28.9 mo. Greater than 25 primary cancers were represented within this retrospective cohort. However, $75 \%$ of cases were accounted for by metastatic lung, breast, prostate, renal, colon, skin, sarcoma, and thyroid cancers. The majority of the tumors $(62 \%)$ localized to the thoracic spine, followed by $25 \%$ lumbar, $11 \%$ cervical, and $1.4 \%$ sacral levels (Table 1). Surgical volume increased over time, with $43 \%$ of the operations performed between 2013 and 2017.

\section{Overall Survival}

The median overall survival for the entire cohort was 8.5 mo (95\% CI 7.6-9.4) and is presented in Table 2 and Figure 1A. An improvement of $1 \%$ with each successive year of surgery was noted in overall survival for the cohort; however, this failed to reach statistical significance (Figure $1 \mathrm{~B}$, Cox model $P=.12$ ). RPA of the entire cohort failed to reveal a split year for survival improvement. Significant differences in survival were observed among primary tumors sites (Figure 1C). Overall, 30-d mortality was $5.5 \%$, and overall, $90-\mathrm{d}$ mortality was $23.7 \%$. There was no statistically significant change in the 30 - or 90 -d mortality over the study time interval $(P=.81$ and $P=.99$, respectively).

\section{Kidney Cancer}

A total of 208 patients (14\%) underwent surgery for the treatment of kidney cancer metastatic to the spine. The median survival for the entire kidney cancer cohort was 11.6 mo (95\% CI: 9.1-13.9). Postoperative survival improved by $3 \%$ (95\% CI: 1\%-6\%) with each successive year of surgery (Cox model $P$-value for association of surgery year with survival $=.01$ ) (Figure 2). Furthermore, RPA revealed that patients who had surgery after 2010 had statistically significantly improved survival compared to patients who had surgery before 2011 , with a $41 \%$ (95\% CI: 20\%-57\%) decreased risk of death if surgery took place after 2010 (Figure 2). Patients who underwent surgery before 2011 had a median survival of 9.4 mo (95\% CI: 6.112.1), whereas patients who had surgery after 2010 had a median 


\begin{tabular}{|c|c|}
\hline Variable & $N=1515$ \\
\hline \multicolumn{2}{|l|}{ Gender } \\
\hline Female & $613(40 \%)$ \\
\hline Male & $902(60 \%)$ \\
\hline Age at index spine mets surgery, mean (range) & $60(18-92)$ \\
\hline \multicolumn{2}{|l|}{ Location } \\
\hline Cervical & $170(11 \%)$ \\
\hline Thoracic & $944(62 \%)$ \\
\hline Lumbar & $380(25 \%)$ \\
\hline Sacrum & $21(1.4 \%)$ \\
\hline \multicolumn{2}{|l|}{ Primary site } \\
\hline Adrenal & $9(0.6 \%)$ \\
\hline Bladder & $33(2.2 \%)$ \\
\hline Brain & $4(0.3 \%)$ \\
\hline Breast & 149 (9.8\%) \\
\hline Cervix & $9(0.6 \%)$ \\
\hline Colon & $99(6.5 \%)$ \\
\hline Esophagus & $17(1.1 \%)$ \\
\hline Gastrointestinal & $48(3.2 \%)$ \\
\hline Head and neck & $45(3.0 \%)$ \\
\hline Kidney & $208(14 \%)$ \\
\hline Liver & $25(1.7 \%)$ \\
\hline Lung & $309(20 \%)$ \\
\hline Lymphoma & $33(2.2 \%)$ \\
\hline Multiple myeloma & $58(3.8 \%)$ \\
\hline Ovary & $6(0.4 \%)$ \\
\hline Pelvis & $14(0.9 \%)$ \\
\hline Penis & $2(0.1 \%)$ \\
\hline Prostate & $148(9.8 \%)$ \\
\hline Sarcoma & $113(7.5 \%)$ \\
\hline Skin & $56(3.7 \%)$ \\
\hline Testicle & $6(0.4 \%)$ \\
\hline Thymus & $4(0.3 \%)$ \\
\hline Thyroid & $59(3.9 \%)$ \\
\hline Unknown or blank in database & $14(0.9 \%)$ \\
\hline Uterus & $47(3.1 \%)$ \\
\hline \multicolumn{2}{|l|}{ Timing } \\
\hline Metachronous & $986(65 \%)$ \\
\hline Synchronous & $529(35 \%)$ \\
\hline \multicolumn{2}{|l|}{ Surgery year } \\
\hline 1998 & $41(2.7 \%)$ \\
\hline 1999 & $37(2.4 \%)$ \\
\hline 2000 & $45(3.0 \%)$ \\
\hline 2001 & $55(3.6 \%)$ \\
\hline 2002 & $35(2.3 \%)$ \\
\hline 2003 & $54(3.6 \%)$ \\
\hline 2004 & 49 (3.2\%) \\
\hline 2005 & $54(3.6 \%)$ \\
\hline 2006 & $56(3.7 \%)$ \\
\hline 2007 & $44(2.9 \%)$ \\
\hline 2008 & $71(4.7 \%)$ \\
\hline 2009 & $59(3.9 \%)$ \\
\hline 2010 & $77(5.1 \%)$ \\
\hline 2011 & $99(6.5 \%)$ \\
\hline 2012 & $84(5.5 \%)$ \\
\hline 2013 & $122(8.1 \%)$ \\
\hline 2014 & $136(9.0 \%)$ \\
\hline 2015 & $132(8.7 \%)$ \\
\hline 2016 & $127(8.4 \%)$ \\
\hline 2017 & $138(9.1 \%)$ \\
\hline
\end{tabular}

survival of 16.3 mo (95\% CI: 10.7-25.9). Furthermore, 90-d postoperative mortality decreased from $17 \%$ to $8 \%$ after 2010 $(P=.049)$, whereas there was no statistically significant change in the 30-d postoperative mortality by RPA-defined time period split.

\section{Breast Cancer}

A total of 149 patients (10\%) underwent surgery for the treatment of breast cancer metastatic to the spine. The median survival for the entire breast cancer cohort was 16.6 mo (95\% CI: 12-24.5). Cox modeling failed to show a statistically significant trend toward improved survival over time annually, though RPA revealed a statistically significant 69\% (95\% CI: 23\%-87\%) decrease in hazard of death among patients who had surgery after 2016 (Figure 3), with the median survival of $14.0 \mathrm{mo}$ (95\% CI: 9.3-20.4) among patients who had surgery prior to 2015. There was no statistically significant difference in the 30and $90-\mathrm{d}$ postoperative mortality for the RPA-defined time period groups.

\section{Lung Cancer}

A total of 309 patients (20\%) underwent surgery for the treatment of lung cancer metastatic to the spine. The median survival for the entire lung cancer cohort was 4.6 mo (95\% CI: $3.7-5.4)$. A $2 \%$ (95\% CI: $0 \%-5 \%$ ) improvement in survival was noted with each progressive year of surgery (Figure 4, Cox model $P$-value for association of surgery year with survival $=.03)$. RPA failed to identify a split in the year of surgery and its association with survival.

\section{Colon Cancer}

A total of 99 patients (7\%) underwent surgery for the treatment of colon cancer metastatic to the spine. The median survival for the entire colon cancer cohort was 7.0 mo (95\% CI: 5.0-8.3). A 5\% (95\% CI: 2\%-9\%) improvement in survival was noted with each progressive year of surgery (Figure 5, Cox model $P$-value for association of surgery year with survival $=.005$ ). RPA failed to identify a split in the year of surgery and its association with survival.

\section{Thyroid, Prostate, Sarcoma, and Skin Cancer}

No significant trends were noted in survival for patients with thyroid, prostate, sarcoma, and skin cancer metastases. RPA for thyroid cancer detected a pattern that was not clinically significant. Table 2 summarizes the survival and mortality from index spine metastasis surgery by primary cancer site.

\section{Metachronous vs Synchronous Metastatic Disease}

Patients who presented with spinal metastases requiring surgery at the time of cancer diagnosis (synchronous) had statistically significantly longer median postoperative survival (11.3 mo, 95\% CI: 9.4-13.4) compared to patients who underwent surgery for metachronous spinal metastases (7.6 mo, 95\%: CI 6.7-8.6, $P<.05$, Figure 6A). Patients undergoing surgery for synchronous 


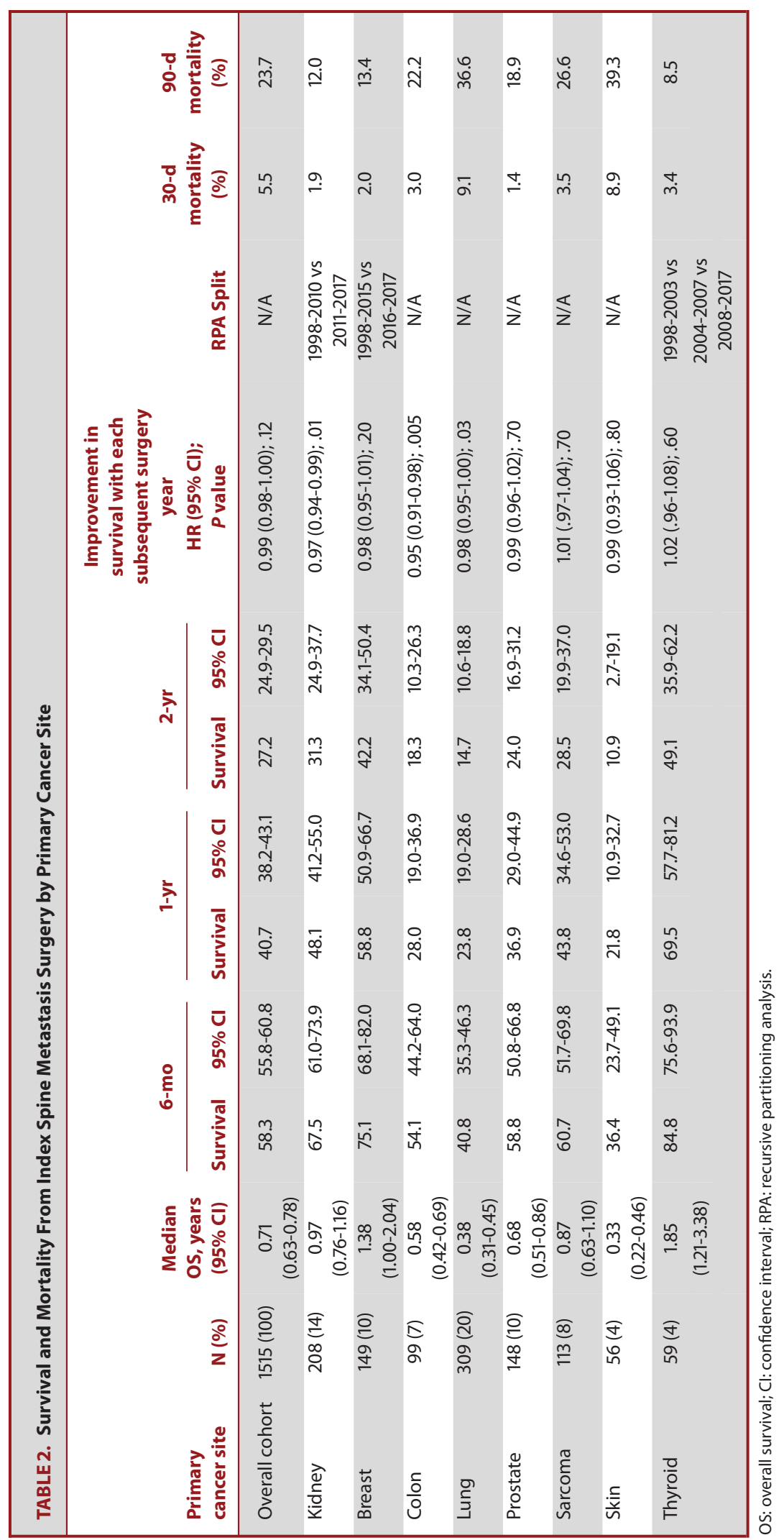




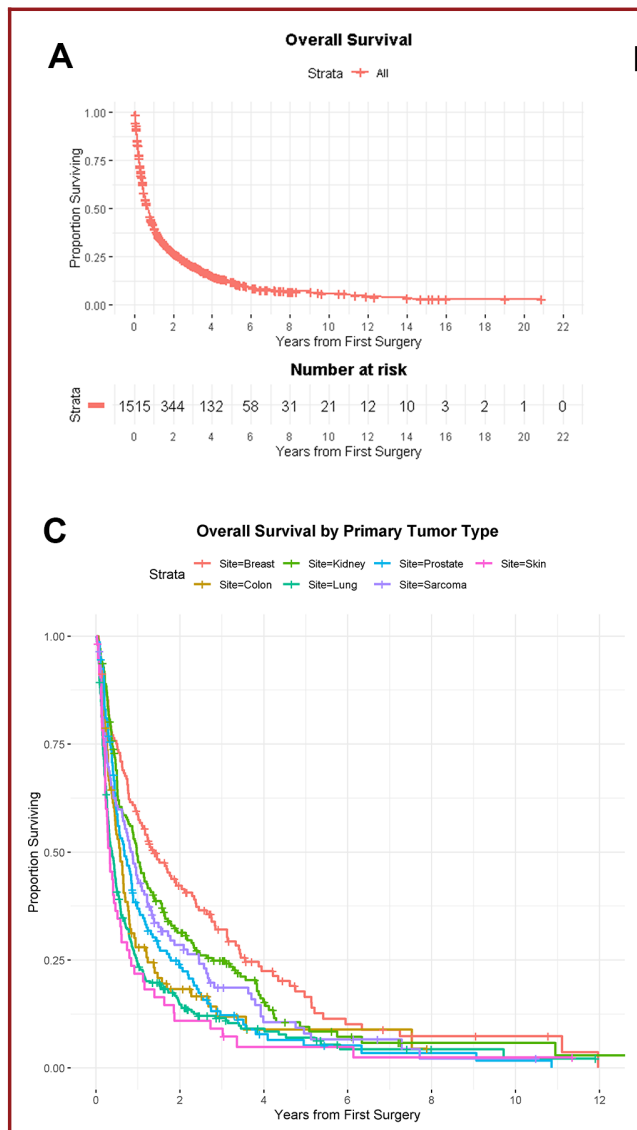

B

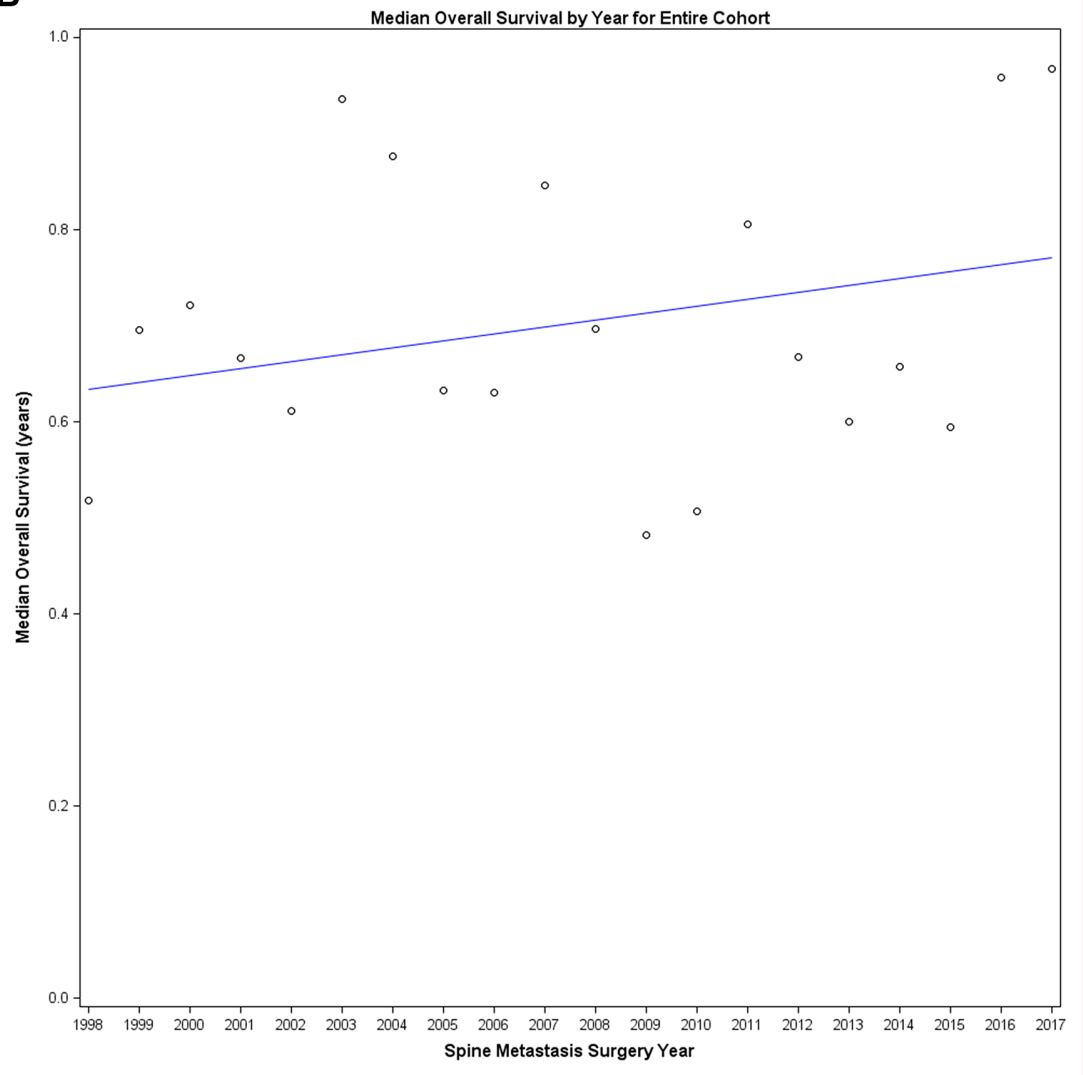

FIGURE 1. A, Overall survival: the median overall survival (OS) for the entire cohort calculated from first surgery was $0.71 \mathrm{yr}(95 \%$ CI: 0.63, 0.78). B, Median survival trend: (Cox model P-value for association of surgery year with survival $=.12$ ). C, Overall survival by primary site.

lung (5.0 mo (95\% CI: 3.7-6.7) vs 4.1 mo (95\% CI: $3.2-$ 5.4), $\log$-rank $P=.02)$ and prostate (20.3 (95\% CI: 5.4-28) vs 7.4 mo (95\% CI: 5.6-9.8), log-rank $P=.03$ ) metastases had statistically significantly better survival compared to patients who required surgery for metachronous metastases. On the other hand, patients with skin cancer metastatic to the spine experienced longer survival if they underwent surgery for the treatment of metachronous metastases (5.2 (95\% CI: 3.4-7.3) vs 2.8 (95\% CI: 1.7-4.1), $\log$-rank $P=.04$ ).

RPA failed to identify a split in the year of surgery and its association with survival for patients with synchronous or metachronous metastases. However, there was a trend toward a 1\% (95\% CI: $0 \%-2 \%)$ improvement in survival with each progressive year of surgery for patients undergoing surgery for metachronous metastases (Cox model $P$-value for association of surgery year with survival $=.06$, Figure $6 \mathrm{~B}$ ).

\section{DISCUSSION}

Advances in cancer detection, systemic therapy, surgery and radiotherapy have increased the duration of survival among cancer patients and the proportion of patients cured of malignant tumors. ${ }^{1}$ The development of new hormone and chemotherapy agents, along with targeted therapy and immunotherapy have been shown to improve survival across nearly all cancer diagnoses. ${ }^{11,12}$ The evolution of radiotherapy techniques has allowed targeted, high-dose radiation treatment and the delivery of ablative radiation doses while sparing the surrounding healthy tissues. ${ }^{13}$ Finally, surgical technology and techniques have also improved with the use of improved intraoperative visualization and navigation, tumor resection methods, functional restoration, and minimally invasive approaches. All of these advances have incrementally improved survival among cancer patients through decreased risk of local recurrence of primary tumors and improved systemic and local control of metastatic disease.

The current analyses traced the change in survival among patients undergoing surgery for the treatment of metastatic spinal tumors in a single specialized cancer center. This provides an opportunity to examine a longitudinal patient population with uniform surgical indications and treatment strategies. The surgical technique for spinal cord decompression and/or stabilization of the spinal column was quite uniform in the 


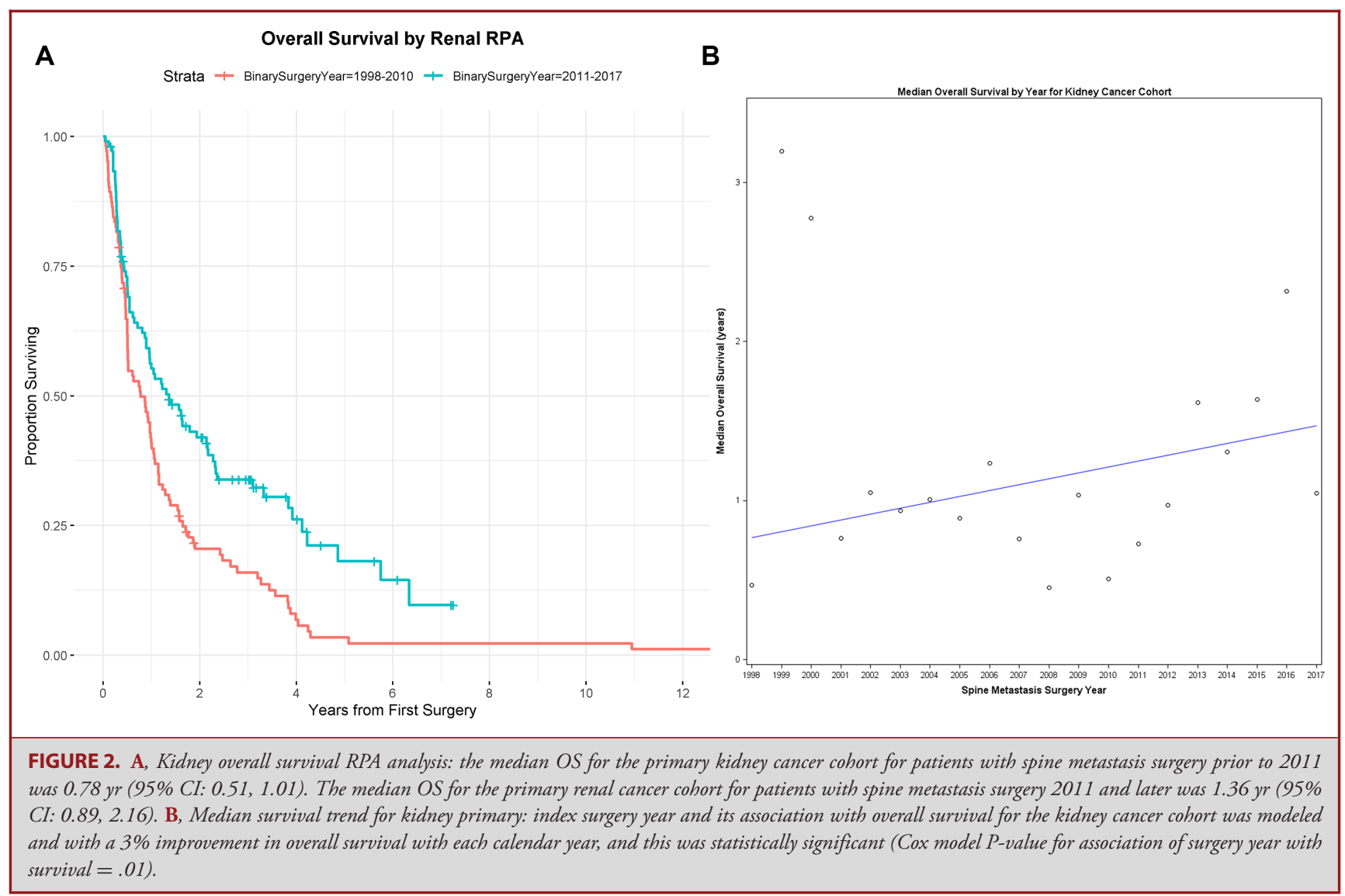

current patient cohort and did not vary with tumor type; therefore, the treatment indications and surgical technique remained uniform over time for the entire study cohort.

Treatment of metastatic spinal tumors serves a palliative function through improvement or preservation of life quality, neurologic function, and spinal stability. Patchell et $\mathrm{al}^{25}$ conducted a prospective randomized trial that demonstrated that for patients with symptomatic solid spinal metastases, surgery followed by radiotherapy provided superior functional outcomes and pain control compared to radiotherapy alone. Furthermore, surgery resulted in greater probability of ambulation restoration, greater duration of ambulation preservation and a small survival advantage. Surgery clearly improves QOL for patients with symptomatic spinal metastases ${ }^{22,26}$ and baseline patient eastern cooperative oncology group, QOL, and neurologic and functional status have been shown to correlate with survival. ${ }^{27}$ Furthermore, there is emerging evidence for survival improvement attributable to ablative therapy for oligometastatic disease. ${ }^{14}$ Thus, although only sparse data support the favorable effect of surgery on survival of patients with spinal metastases, surgery may extend survival through improved function and facilitation of ablative focal therapy for patients with oligometastatic disease.

\section{Key Results}

In the current cohort, the survival changes over time are likely attributable to systemic therapy improvement and reflect the improvement in primary tumor-specific survival. Primary sitespecific analysis showed notable improvement for a few cancers over the study period. Our data indicate that patients who underwent surgery for kidney tumors metastatic to the spine experienced a $60 \%$ improvement in survival. This mirrors the large survival improvement for the whole kidney cancer patient population, in which the 5-yr survival improved from 57\% to $74 \%$ when comparing the 1987 to 1989 time interval to the 2006 to 2012 interval. Although the majority of the improvement is due to early detection, drugs targeting the VEGF pathway, mammalian target of rapamycin inhibitors, and immune checkpoint inhibitors have also contributed to improved survival for patients with clear-cell renal-cell carcinoma. ${ }^{15}$

In the current cohort, patients who underwent spinal surgery for lung cancer metastases experienced a $40 \%$ improvement in survival. In the general lung cancer patient population, the 5-yr relative survival has also improved from $12 \%$ in 1975 to 1977 to $20 \%$ in 2009 to 2015 . $^{2}$ A significant proportion of patients with nonsmall cell lung carcinoma have targetable driver mutations and benefited from advances in systemic therapy, 


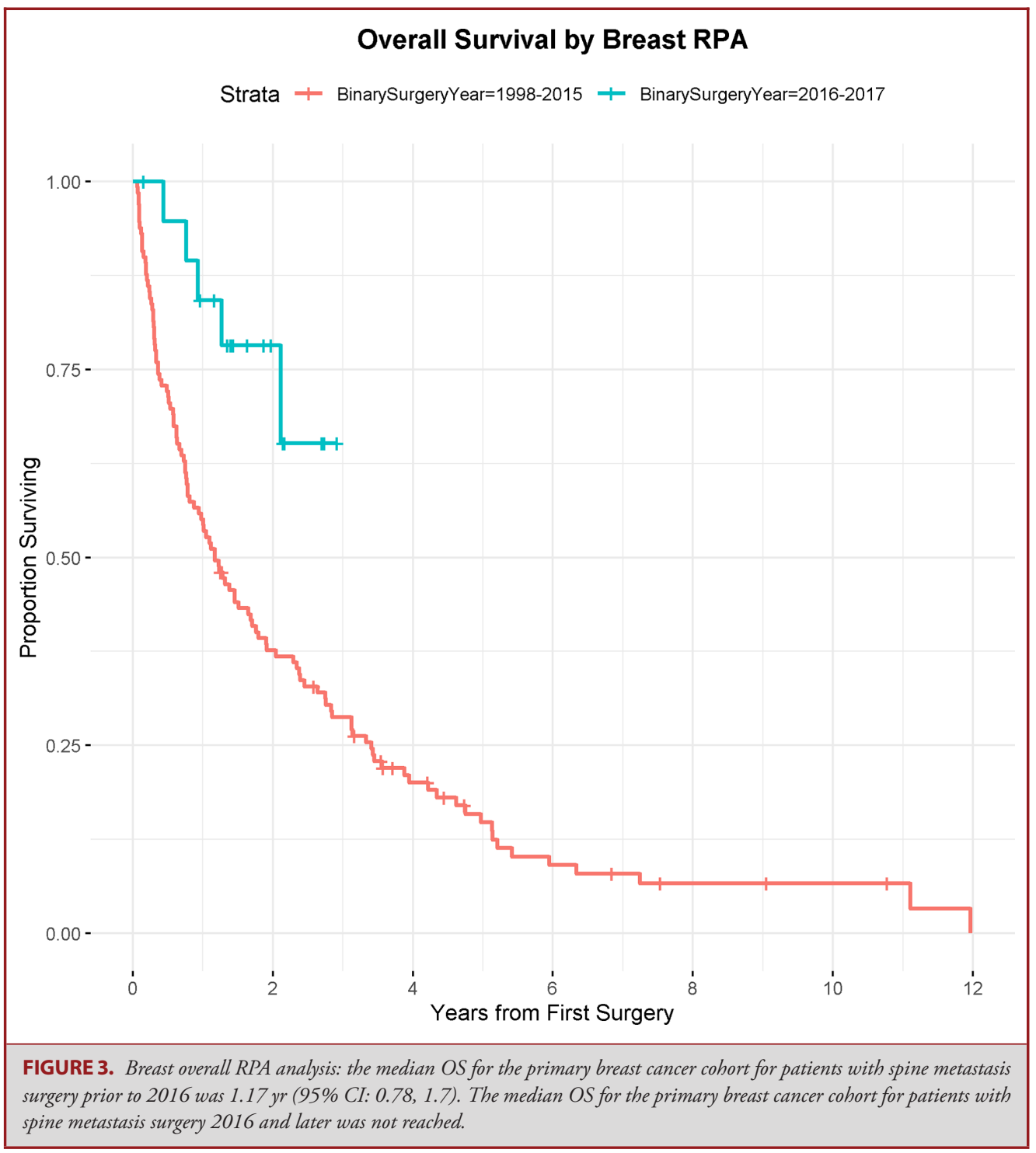

with tyrosine kinase inhibitors targeting the epidermal growth factor receptor and activin like kinase alterations and immune checkpoint inhibitors having been shown to extend survival. ${ }^{16}$ However, although the use of targeted treatments has increased among patients with metastatic nonsmall-cell lung carcinoma, SEER data indicate that the survival improved by only $1.5 \mathrm{mo}$ from 2000 to $2010 .{ }^{17}$ Furthermore, 57\% of lung cancer patients present with distant metastases at the time of initial diagnosis and have a short 5 -yr relative survival of $5 \%$. These data are consistent with the current analysis, in which we found that in spite of relative improvement in postoperative survival, the median postoperative survival for lung cancer patients remained fairly short.

Patients with colon cancer doubled their postoperative survival over our study period. Similarly, observation of survival trends for the general colon cancer patient population showed that patients experienced a significant improvement in survival over the span from 1970 to 2004 . The 1-yr survival improved from $17 \%$ to $24 \%$ among men with Stage IV colon cancer and from $23 \%$ to $46 \%$ among women. ${ }^{18}$ This improvement is thought to be attributable to increased use of systemic chemotherapy and excision of liver metastases.

Thus, the improvements in postoperative survival for patients with kidney, lung, and colon cancers metastatic to the spine show changes similar to the survival improvements in the general patient population with these cancers. Although the annual overall survival improvement for the patient population was modest at $1 \%$ per year, this amounted to a $20 \%$ improvement in survival over the study period. The heterogeneity of survival changes across all primary tumor groups likely explains the lack of 


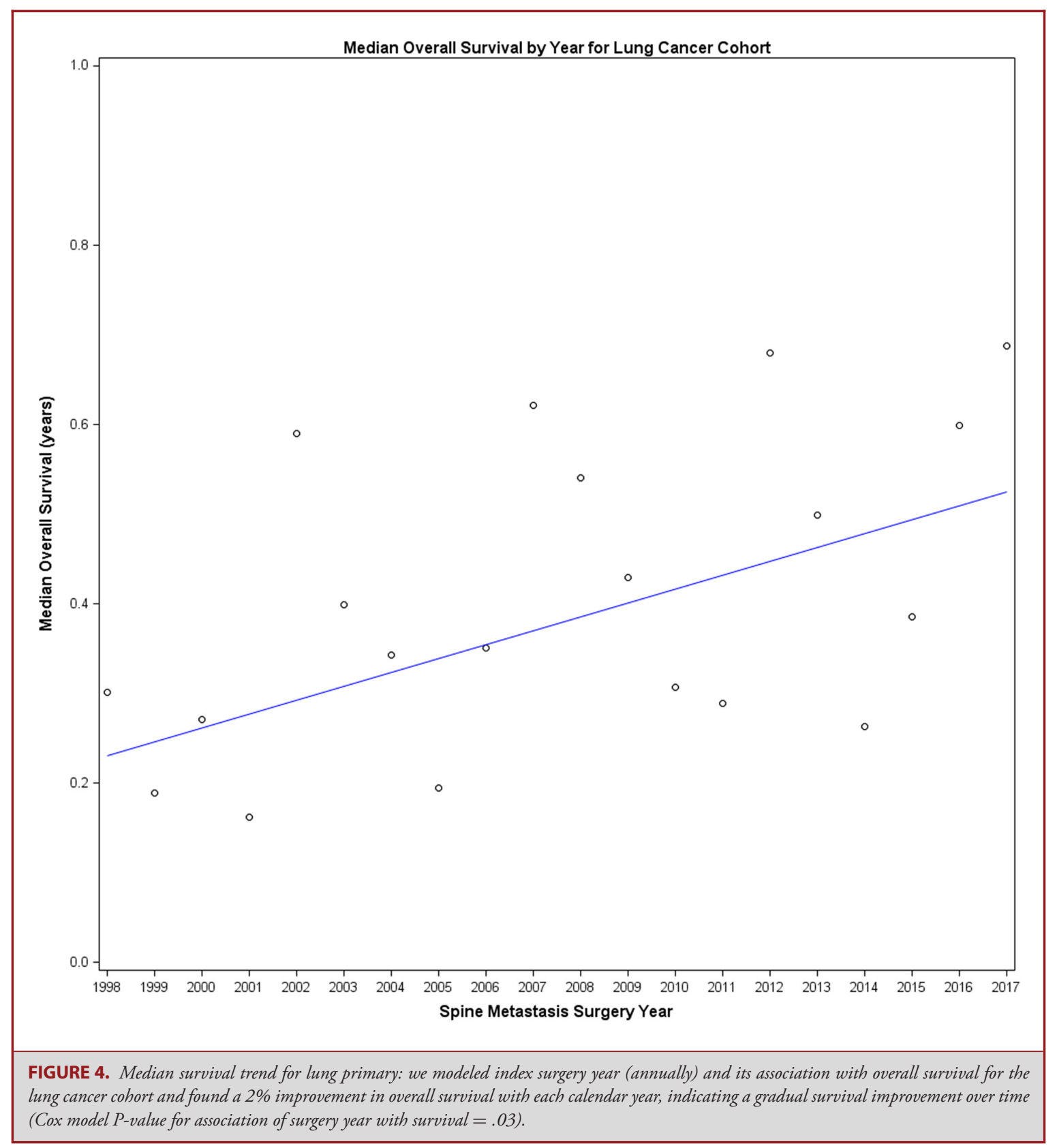

statistical significance for this change. Overall, the survival among patients with metastatic cancer has improved over time, albeit the changes remain modest.

\section{Interpretation}

Technological advances in genetic and molecular analysis have greatly improved our understanding of mutations responsible for the development and progression of cancer and provided opportunities to target specific cancer drivers. However, with over 3000 uniquely identified mutations, only $7.5 \%$ of patients derive clinical benefit from targeted therapies. ${ }^{11}$ Significant work has been done in order to delineate patient-specific markers of survival, including targets for tyrosine kinase inhibitors, monoclonal antibodies, and hormonal therapy, which have been shown to be predictors of survival after surgery for spinal metastases. ${ }^{19-21}$ Unfortunately, cancer patients with targetable tumor drivers represent only a small segment of this patient population. Thus, even in the age of targeted therapy, primary tumor site remains a significant predictor of survival, and the current analysis confirms this, with significant differences in the median survival observed among patients with different primary tumors. 


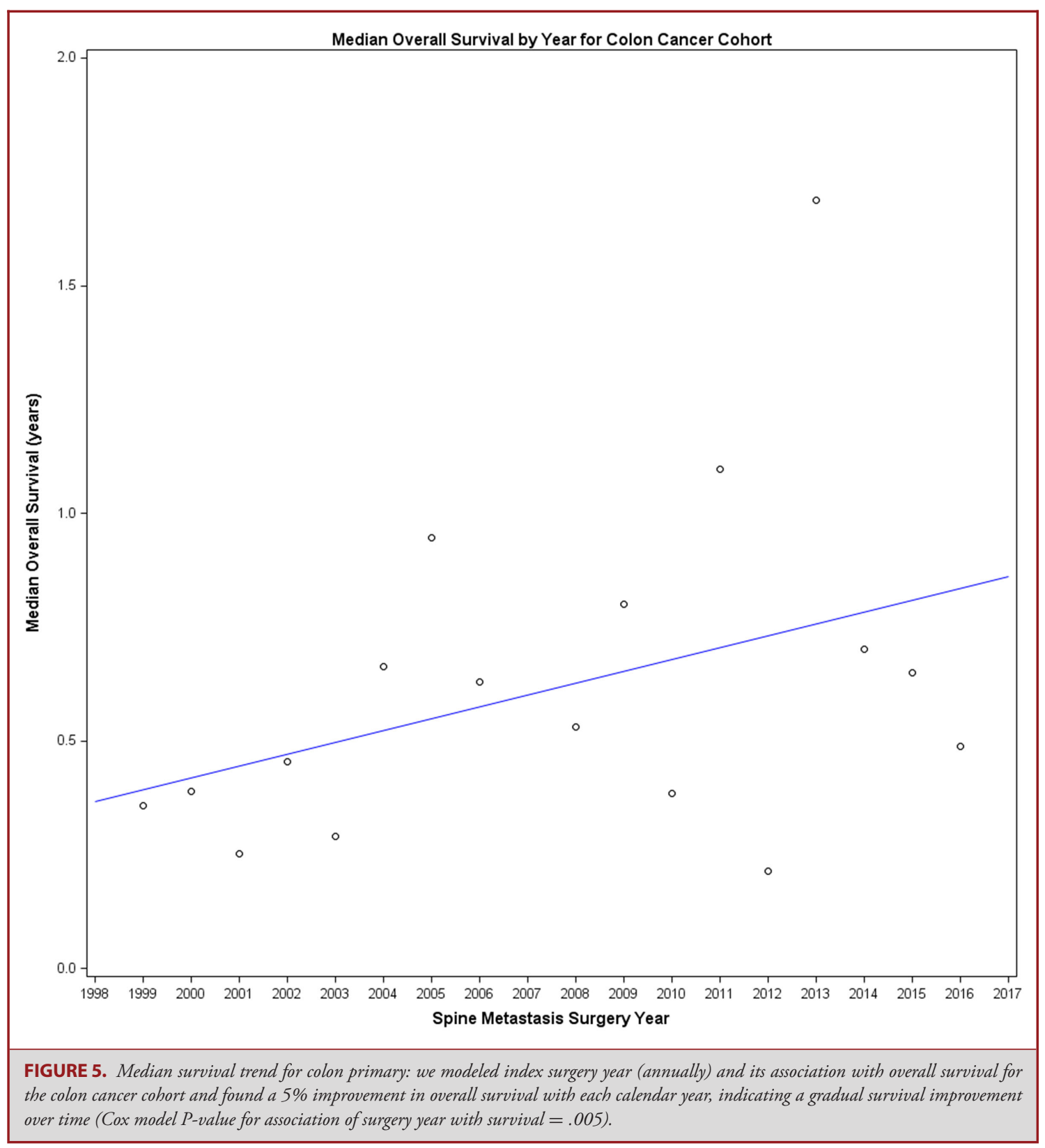

RPA identified splits by surgery period and the association with postoperative survival for patients with kidney and breast tumors metastatic to the spine, with recently treated patients surviving longer. The years of the splits do not seem to correlate to clear changes in systemic therapy; however, there may be a lag between the introduction of new agents and survival improvement in patient populations. Although establishment of the causation of the splits is beyond the scope of the current analysis, they are likely attributable to improvement in the systemic therapy options, more effective local treatment options, and additional options that can be offered for salvage of progressive and recurrent disease. The slight improvement in the survival of patients undergoing surgery for the treatment of metachronous spinal metastases supports the possibility of additional effective systemic lines of therapy available to patients presenting with tumor progression. These splits and overall survival improvement trends serve as interesting pilot data for further studies for molecular and therapeutic correlates of survival improvement in patients with spinal metastases.

Analysis of the 30- and 90-d postoperative mortality trends failed to show significant changes over time, except for patients with kidney cancer. These data emphasize the significant risk 


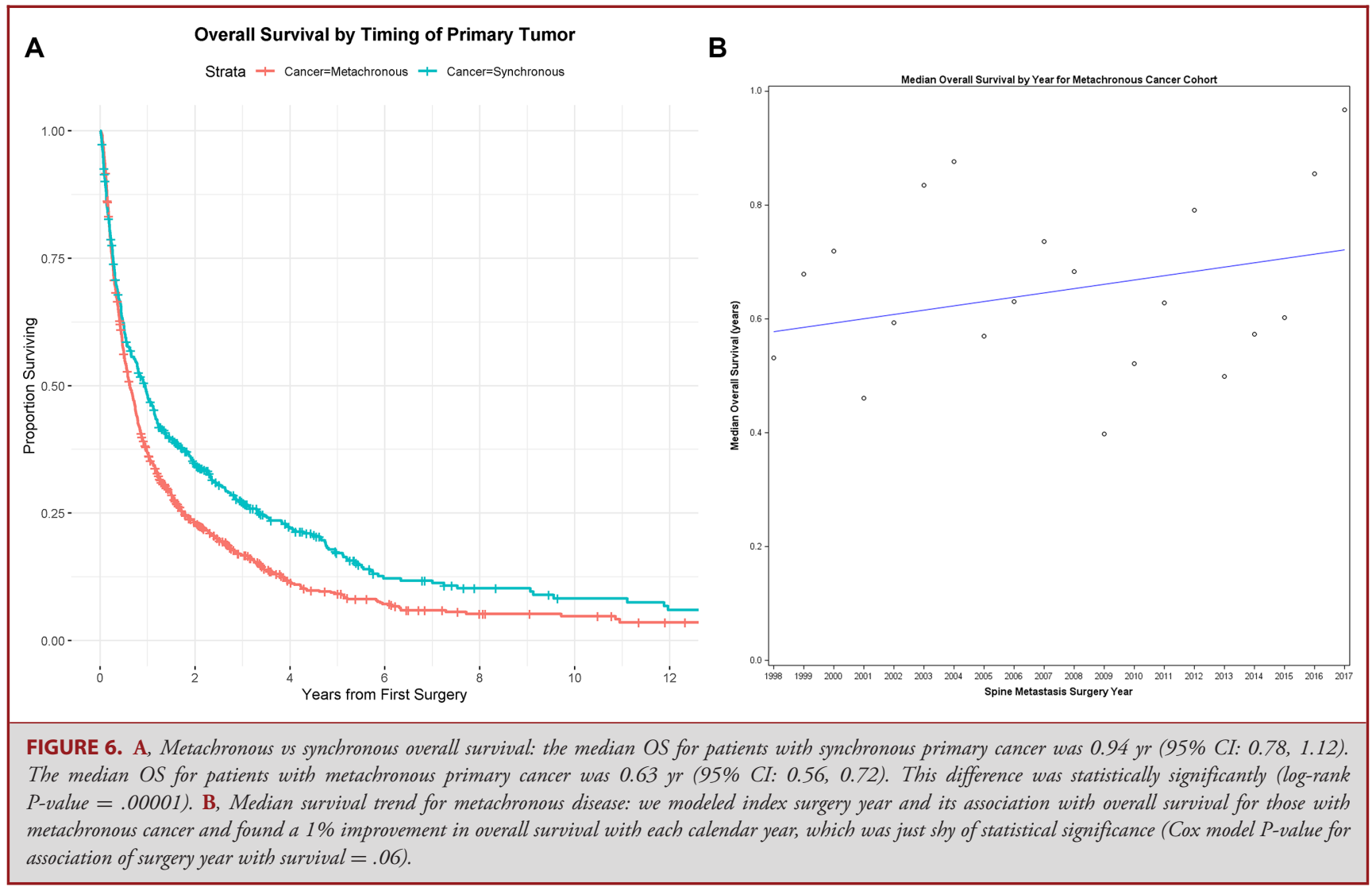

profile of patients undergoing surgery for the treatment of spinal metastases. Fehlings et $\mathrm{al}^{22}$ reported a $9.1 \% 30-\mathrm{d}$ and $45.5 \%$ 90-d mortality in the prospective AOSpine cohort, which is in line with the current data. Instruments such as the Skeletal Oncology Research Group (SORG) nomogram, the Modified Tokuhashi score, and the New England Spinal Metastasis Score (NESMS) provide validated and accurate postoperative survival prediction for patients with spinal metastases, and identification of patients at the extremes of the survival curves might significantly alter treatment decisions. ${ }^{23}$ However, the impact of survival prediction instruments on clinical decisions requires further elucidation because the decision to take the patient to surgery requires consideration of multiple factors besides the expected survival, such as the goals of the patient, the expected impact of surgery on the patient's QOL, and the invasiveness of the operation. ${ }^{24}$

\section{Limitations}

The current analysis has several limitations. The study includes a heterogeneous population of patients with numerous tumor histologies undergoing a wide range of systemic therapies. Furthermore, although the current study classifies patients according to the primary tumor site, further classification of tumors based on pathologic diagnosis, genetics, and molecular profile was not performed in the current analysis. We plan future studies that will focus on specific primary tumors from this cohort in order to analyze the possible associations between survival and specific molecular and genetic markers and systemic therapy. Because of the myriad of systemic treatments available, we could not effectively control for this factor in such a large patient population and such an extended time interval. Furthermore, the temporal trends in the QOL and neurological outcomes were not examined because of the lack of patient reported outcome collection prior to 2012.

\section{Generalizability}

The single-center outcomes from a cancer center may not be generalizable to other medical entities. Utilization of national healthcare databases and analysis of survival in various medical health care setting should be utilized in order to determine whether similar survival changes occurred in broader patient populations.

\section{CONCLUSION}

Significant developments in oncology have extended survival for patients with cancer. The current analysis illustrates that over the past $20 \mathrm{yr}$, the survival among patients with lung, renal, and colon cancer undergoing surgery for the treatment of spinal metastases has also significantly improved. These data support the need for durable systemic and local treatment strategies for 
patients with spinal metastases, and for outcome studies with emphasis on long-term QOL and return to work.

\section{Funding}

This project was funded in part with support from the National Institutes of Health core grant ( P30 CA008748).

\section{Disclosures}

The authors have no personal, financial, or institutional interest in any of the drugs, materials, or devices described in this article. Dr Laufer's disclosures are the following: consulting for Depuy/Synthes, Medtronic, Spinewave, Brainlab, and Globus.

\section{REFERENCES}

1. Klimo P Jr, Thompson CJ, Kestle JRW, Schmidt MH. A meta-analysis of surgery versus conventional radiotherapy for the treatment of metastatic spinal epidural disease. Neuro Oncol. 2005;7(1):64-76.

2. U.S. Mortality Data 1969-2016 when Using SEER*Stat: Surveillance, Epidemiology, and End Results (SEER) Program SEER*Stat Database: Mortality-All COD, Aggregated With State. SEER. Accessed February 11, 2020.

3. Kummar S, Chen HX, Wright J, et al. Utilizing targeted cancer therapeutic agents in combination: novel approaches and urgent requirements. Nat Rev Drug Discov. 2010;9(11):843-856.

4. Richards L, Misra V, Verma R, Greenhalgh S. Metastatic spinal cord compression (MSCC) - Collaborative Work between the tertiary cancer centre and the specialist spinal centre since the introduction of the MSCC coordinator service has seen a marked increase in surgical rates, with $20 \%$ of patients who presented with MSCC in the first 24 months having spinal surgery. This has resulted in improved survival rates for MSCC patients in greater manchester and cheshire. Spine J. 2017;17(3):S30-S31.

5. Laufer I, Iorgulescu JB, Chapman T, et al. Local disease control for spinal metastases following "separation surgery" and adjuvant hypofractionated or highdose single-fraction stereotactic radiosurgery: outcome analysis in 186 patients. J Neurosurg Spine. 2013;18(3):207-214.

6. Bate BG, Khan NR, Kimball BY, Gabrick K, Weaver J. Stereotactic radiosurgery for spinal metastases with or without separation surgery. J Neurosurg Spine. 2015;22(4):409-415.

7. Barzilai O, Amato M-K, McLaughlin L, et al. Hybrid surgery-radiosurgery therapy for metastatic epidural spinal cord compression: a prospective evaluation using patient-reported outcomes. Neurooncol Pract. 2018;5(2):104-113.

8. Barzilai O, McLaughlin L, Amato M-K, et al. Predictors of quality of life improvement after surgery for metastatic tumors of the spine: prospective cohort study. Spine J. 2018;18(7):1109-1115.

9. Laufer I, Rubin DG, Lis E, et al. The NOMS framework: approach to the treatment of spinal metastatic tumors. Oncologist. 2013;18(6):744-751.

10. Therneau TM. Extending the cox model. Proc First Seattle Symp Biostat. 1997:5184 (doi:10.1007/978-1-4684-6316-3_5).

11. Chakravarty D, Gao J, Phillips SM, et al. OncoKB: a precision oncology knowledge base. JCO Precis Oncol. 2017;2017:http://dx.doi.org/10.1200/PO.17.00011.
12. Miller KD, Nogueira L, Mariotto AB, et al. Cancer treatment and survivorship statistics, 2019. CA A Cancer J Clin. 2019;69(5):363-385.

13. Vellayappan BA, Chao ST, Foote M, et al. The evolution and rise of stereotactic body radiotherapy (SBRT) for spinal metastases. Expert Rev Anticancer Ther. 2018;18(9):887-900.

14. Iyengar P, Wardak Z, Gerber DE, et al. Consolidative radiotherapy for limited metastatic non-small-cell lung cancer: a phase 2 randomized clinical trial. JAMA Oncol. 2018;4(1):e173501.

15. Choueiri TK, Motzer RJ. Systemic therapy for metastatic renal-cell carcinoma. N Engl J Med. 2017;376(4):354-366.

16. Salehi-Rad R, Li R, Paul MK, Dubinett SM, Liu B. The biology of lung cancer: development of more effective methods for prevention, diagnosis, and treatment. Clin Chest Med. 2020;41(1):25-38.

17. Bradley JD, Hu C, Komaki RU, et al. Long-Term results of RTOG 0617: a randomized phase 3 comparison of standard dose versus high dose conformal chemoradiation therapy $+1-$ Cetuximab for stage III NSCLC. Int I Radiat Oncol Biol Phys. 2017;99(2):S105.

18. Snaebjornsson P, Jonasson L, Olafsdottir EJ, et al. Why is colon cancer survival improving by time? A nationwide survival analysis spanning 35 years. Int J Cancer. 2017;141(3):531-539.

19. Sciubba DM, Goodwin CR, Yurter A, et al. A systematic review of clinical outcomes and prognostic factors for patients undergoing surgery for spinal metastases secondary to breast cancer. Global Spine J. 2016;6(5):482-496.

20. Ahangar P, Aziz M, Rosenzweig DH, Weber MH. Advances in personalized treatment of metastatic spine disease. Ann Transl Med. 2019;7(10):223-223.

21. Choi BD, Shankar GM, Sivaganesan A, Van Beaver LA, Oh K, Shin JH. Implication of biomarker mutations for predicting survival in patients with metastatic lung cancer to the spine. Spine. 2018;43(21):E1274-E1280.

22. Fehlings MG, Nater A, Tetreault L, et al. Survival and clinical outcomes in surgically treated patients with metastatic epidural spinal cord compression: results of the prospective multicenter AOSpine study. J Clin Oncol. 2016;34(3):268-276.

23. Pereira NRP, Janssen S, Ferrone M, Schwab JH. Development of a prognostic survival algorithm for patients with metastatic spine disease. Spine J. 2016; 16(10):S318.

24. Barzilai O, Fisher CG, Bilsky MH. State of the art treatment of spinal metastatic disease. Neurosurgery. 2018;82(6):757-769.

25. Patchell RA, Tibbs PA, Regine WF, et al. Direct decompressive surgical resection in the treatment of spinal cord compression caused by metastatic cancer: a randomised trial. Lancet North Am Ed. 2005;366(9486):643-648.

26. Hussain I, Barzilai O, Reiner AS, McLaughlin LA, Bilsky MH, Laufer I. Validation of spinal instability neoplastic score components through patientreported outcomes. Spine J. 2017;17(10):S181.

27. Falicov A, Fisher CG, Sparkes J, Boyd MC, Wing PC, Dvorak MF. Impact of surgical intervention on quality of life in patients with spinal metastases. Spine. 2006;31(24):2849-2856

Supplemental digital content is available for this article at www. neurosurgery-online.com.

Supplemental Digital Content. Figure. Patient selection flow chart. 